\title{
Unearthing wave-function renormalization effects in the time evolution of a Bose-Einstein condensate
}

\author{
Paolo Facchi ${ }^{1}$, Saverio Pascazio ${ }^{1}$, Francesco V. Pepe ${ }^{1}$, Ennio \\ Arimondo $^{2,3}$, Donatella Ciampini ${ }^{2,3}$ \& Oliver Morsch ${ }^{3}$ \\ ${ }^{1}$ Dipartimento di Fisica and MECENAS, Università di Bari, I-70126 Bari, Italy \\ and INFN, Sezione di Bari, I-70126 Bari, Italy \\ ${ }^{2}$ CNISM-Pisa, Dipartimento di Fisica, Università di Pisa, Lgo Pontecorvo 3, 56127 \\ Pisa, Italy \\ ${ }^{3}$ INO-CNR, Dipartimento di Fisica, Università di Pisa, Lgo Pontecorvo 3, I-56127 \\ Pisa,Italy
}

\begin{abstract}
We study the time evolution of a Bose-Einstein condensate in an accelerated optical lattice. When the condensate has a narrow quasimomentum distribution and the optical lattice is shallow, the survival probability in the ground band exhibits a steplike structure. In this regime we establish a connection between the wave-function renormalization parameter $Z$ and the phenomenon of resonantly enhanced tunneling.

PACS numbers: 03.75.Lm, 03.65.Xp, 11.10.Gh
\end{abstract}

\section{Introduction}

Renormalization effects are among the most subtle and interesting in quantum field theory [1] and go to the heart of our understanding of fundamental interactions. Free field operators create and annihilate single particles with unit probability. The presence of interactions makes things much more complicated and requires powerful analytical tools, in order to compute the propagator and the $S$-matrix through the self-energy function and guarantee probability conservation. In the Källén-Lehmann representation [2], probability conservation is enforced via wave function renormalization, that consists in a rescaling of quantum fields by a factor $\sqrt{Z}$ to take into account the effects of interactions.

The propagator yields exponential decay (Fermi "golden" rule) due to the contribution of a pole on the second Riemann sheet in the complex energy plane [3], but gets also a contribution of order (coupling constant) $)^{2}$ from a contour integration in the complex energy plane, that modifies the exponential decay law both at short and long times, yielding the characteristic quadratic and power-law behaviors. The quantity $\sqrt{Z}$ is the modulus of the residue of a pole of the propagator in the second Riemann sheet in the complex energy plane and is different from unity when the latter is computed 


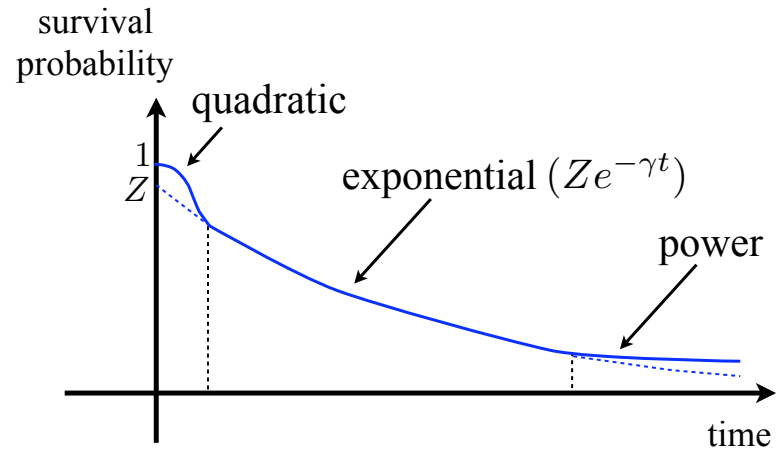

Figure 1. Survival probability of an unstable system. The exponential decay law $P(t) \simeq P_{\mathrm{Z}}(t)=Z e^{-\gamma t}$ is only valid at intermediate times and its extrapolation at $t=0$ yields wave function renormalization $Z$. In general $Z \neq 1$.

beyond leading order in the coupling constant. For a stable state one has $Z<1$ (due to probability conservation in the Källén-Lehmann representation), but for an unstable state $Z$ is unconstrained and can be $>1$ [4]. This is a common feature of quantum decay processes arising from the coupling of a discrete level to a continuum. In such a case the discrete level moves away from the real axis and acquires an imaginary part, which accounts for its decay rate. Since $Z$ is the square of the overlap between the initial state and this non-normalizable complex-energy vector, known as "Gamow state" [5, 6, 7, one can have $Z>1$.

A typical time evolution of the survival probability of an unstable system in its initial state is displayed in Fig. 1. The exponential law is approximately valid in an intermediate time region. It is preceded by a quadratic decay (Zeno region) and superseded by a power law. Notice that the extrapolation of the exponential to $t=0$ yields a value (wave function renormalization $Z$ ) that is in general $\neq 1$. We shall write

$$
P(t)=Z e^{-\gamma t}+\text { additional contributions, }
$$

where the additional contributions are typically second order in the coupling (to a continuum of states to which the system decays) and are important at short and long times. At intermediate times the exponential is assumed to dominate and characterize the evolution

$$
P(t) \simeq P_{\mathrm{Z}}(t)=Z e^{-\gamma t},
$$

with $\gamma$ given by the Fermi golden rule.

The short-time quadratic (and hence non-exponential) decay was first experimentally observed by the group led by Mark Raizen in Texas [8, 9]. In this pioneering work focus was on the occurrence of the quantum Zeno effect and its inverse [10, 11] due to repeated measurements. In this article we shall focus on the same physical system used in [8, 9, but under very different physical conditions, both in terms of initial state and physical parameters [12]. This will enable us to unearth wave-function renormalization effects. 


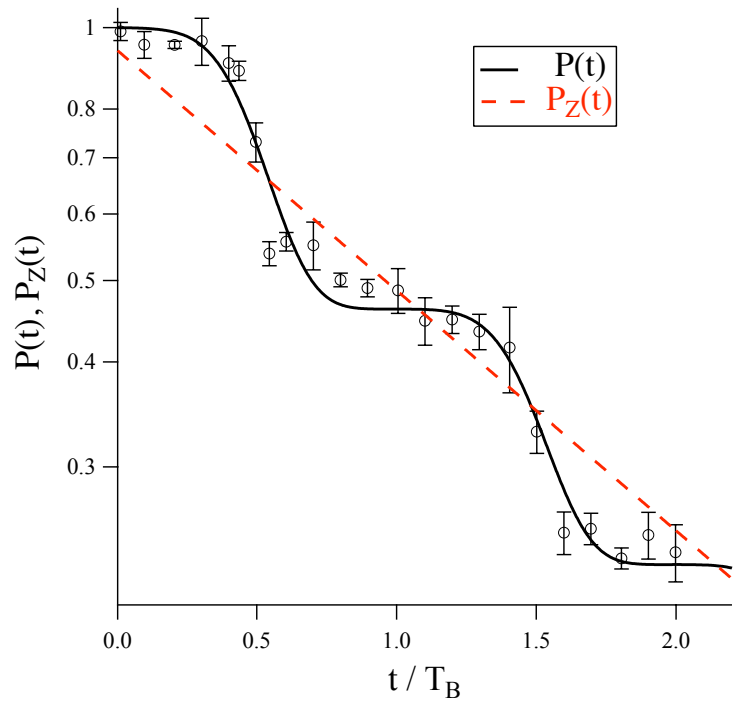

Figure 2. Survival probability $P(t)$ of the wave packet of the atomic cloud in the lowest energy band of the accelerated optical lattice. The numerical solution of the Schrödinger equation is the full line. The experimental data (open circles) are the same as in Ref. [12] and the (red) dashed line is the exponential fit based on $P_{\mathrm{Z}}(t)$ in Eq. (35), whose crossing with the $y$ axis yields the value of $Z$. The slope of the exponential decay yields the decay rate $\gamma$ (Fermi Golden rule). In this plot $V_{0}=1 E_{\text {rec }}, F_{0}=0.383$, yielding $Z<1$.

Let us briefly sketch the main ideas to be explored in this note. We shall investigate the time evolution of a wave packet (a "cloud" of atoms in a Bose-Einstein condensate) in a tilted optical lattice. The wave packet, initially in the lowest energy band, is narrow in momentum space and feels a constant acceleration due to the lattice tilt. When it reaches the edge of the band, it can make a Landau-Zener transition [13] to the upper band. The survival probability in the lowest energy band is therefore characterized by plateaus (when the packet evolves in the band) and sharp transition regions (when the packet loses probability to the upper band). A typical example is displayed in Fig. 2. One clearly sees that in practice, the dynamics can be rather different from the idealized situation depicted in Fig. 1. The overall process is involved and the quantum dynamics depends on a plethora of factors, ranging from probability losses to phase coherence effects. We shall see that Landau-Zener transitions [13] and Stückelberg's oscillations [14 are two facets of this complex evolution and that by scrutinizing the features of the survival probability of the wave function that collectively describes a cold atomic cloud, one can extract crucial information on wave-function renormalization effects.

In this article we shall endeavor to give an elementary introduction to this problem and discuss in more detail the model adopted in Ref. [12] to study these effects. We shall also scrutinize the convergence of the decay rate and wave-function renormalization parameter to their asymptotic values. The main idea will be to use Landau-Zener transitions as a benchmark for the study of wave-function renormalization effects. 
Unearthing wave-function renormalization effects in the time evolution of a Bose-Einstein condensate4

\section{Landau-Zener tunneling}

A Landau-Zener (LZ) transition takes place in a two-level system with a time-dependent Hamiltonian $H_{\mathrm{LZ}}(t)$ whose spectrum, as a function of time, is characterized by the presence of an avoided crossing [13, 14, 15, 16]. In the time-independent, diabatic basis $\{|1\rangle,|2\rangle\}$, the expectation values of the Hamiltonian are linear in time

$$
\left\langle 1\left|H_{\mathrm{LZ}}(t)\right| 1\right\rangle=-\left\langle 2\left|H_{\mathrm{LZ}}(t)\right| 2\right\rangle=-\alpha t, \quad(\alpha>0)
$$

while the off-diagonal matrix elements are constant in time and can be assumed to be real by properly adjusting the phase of the basis states

$$
\left\langle 1\left|H_{\mathrm{LZ}}(t)\right| 2\right\rangle=\left\langle 2\left|H_{\mathrm{LZ}}(t)\right| 1\right\rangle=\delta .
$$

The state $|\psi(t)\rangle$ of the system is expanded as

$$
|\psi(t)\rangle=a_{1}(t)|1\rangle+a_{2}(t)|2\rangle
$$

and the Schrödinger equation reads

$$
i \hbar \frac{d}{d t}\left(\begin{array}{l}
a_{1} \\
a_{2}
\end{array}\right)=\left(\begin{array}{cc}
-\alpha t & \delta \\
\delta & \alpha t
\end{array}\right)\left(\begin{array}{l}
a_{1} \\
a_{2}
\end{array}\right) .
$$

The time-dependent eigenbasis of $H_{\mathrm{LZ}}$ is called adiabatic basis and can be obtained by a rotation

$$
\left(\begin{array}{l}
|1(t)\rangle \\
|2(t)\rangle
\end{array}\right)=\left(\begin{array}{rr}
\cos \theta(t) & -\sin \theta(t) \\
\sin \theta(t) & \cos \theta(t)
\end{array}\right)\left(\begin{array}{l}
|1\rangle \\
|2\rangle
\end{array}\right)
$$

with $\theta(t)=\arctan (\alpha t / \delta) / 2$. The adiabatic vectors satisfy

$$
H_{\mathrm{LZ}}(t)|1(t)\rangle=-\Omega(t)|1(t)\rangle, \quad H_{\mathrm{LZ}}(t)|2(t)\rangle=\Omega(t)|2(t)\rangle,
$$

with

$$
\Omega(t)=\sqrt{(\alpha t)^{2}+\delta^{2}}
$$

The degeneration of the mean energy of the diabatic states at $t=0$ is reflected in an avoided energy level crossing in the adiabatic basis, with the two states reaching the minimum energy distance at $t=0$. By setting

$$
|\psi(t)\rangle=b_{1}(t)|1(t)\rangle+b_{2}(t)|2(t)\rangle,
$$

from

we get

$$
\frac{d}{d t}|1(t)\rangle=-\dot{\theta}(t)|2(t)\rangle, \quad \frac{d}{d t}|2(t)\rangle=+\dot{\theta}(t)|1(t)\rangle
$$

$$
i \hbar \frac{d}{d t}\left(\begin{array}{l}
b_{1} \\
b_{2}
\end{array}\right)=\left(\begin{array}{cc}
-\Omega(t) & -i \hbar \dot{\theta}(t) \\
i \hbar \dot{\theta}(t) & \Omega(t)
\end{array}\right)\left(\begin{array}{l}
b_{1} \\
b_{2}
\end{array}\right) .
$$

Therefore, in the adiabatic basis, the evolution of the system is governed by the Hamiltonian

$$
H_{\mathrm{ad}}(t)=-\Omega(t) \sigma_{z}+\Gamma(t) \sigma_{y}, \quad \Gamma(t)=\hbar \dot{\theta}(t)=\frac{\hbar \alpha \delta}{2 \Omega^{2}(t)},
$$


with $\sigma_{y}$ and $\sigma_{z}$ the Pauli matrices. It is clear that the nature of the coupling in the two bases is very different: the coupling between diabatic states is constant in time, while the adiabatic states are significantly coupled only near $t=0$, the off-diagonal terms in (13) being inversely proportional to the square of the distance between the adiabatic levels $2 \Omega(t)$. This enables one to simplify the description of the LZ transition in the adiabatic basis. As a useful approximation, we can replace the Hamiltonian (13) in an interval of width $T_{\mathrm{LZ}} \sim \delta / \alpha$ around $t=0$ with one with constant coefficients

$$
H_{\mathrm{ad}}(t) \simeq H_{\mathrm{ad}}(0)=-\delta \sigma_{z}+\frac{\hbar \alpha}{2 \delta} \sigma_{y}, \quad\left(|t| \lesssim T_{\mathrm{LZ}} / 2\right)
$$

and assume that outside this time interval the adiabatic states evolve uncoupled, $H_{\mathrm{ad}}(t) \simeq-\alpha t \sigma_{z}$.

The afore-mentioned transition time $T_{\mathrm{LZ}}$ can be fixed by imposing that the probability that the system, prepared in state $|1(t)\rangle$ at $t \rightarrow-\infty$, evolves into $|2(t)\rangle$ at $t \rightarrow+\infty$, be given by the Landau-Zener transition probability

$$
P_{\mathrm{LZ}}=\exp \left(-\frac{\pi \delta^{2}}{\hbar \alpha}\right)
$$

Moreover, the $\sigma_{z}$ term in the Hamiltonian (14) can be neglected with good approximation provided

$$
\frac{\Omega(0)}{\Gamma(0)}=\frac{2 \delta^{2}}{\hbar \alpha}=-\frac{2}{\pi} \ln P_{\mathrm{LZ}}
$$

be sufficiently small. Within this approximation, the (unitary) evolution in the interval $\left(-T_{\mathrm{LZ}} / 2, T_{\mathrm{LZ}} / 2\right)$ is governed by

$$
U=\exp \left(-i \frac{\hbar \alpha}{\delta} \frac{T_{\mathrm{LZ}}}{2} \sigma_{y}\right)=\left(\begin{array}{cc}
\cos \left(\hbar \alpha T_{\mathrm{LZ}} / 2 \delta\right) & -\sin \left(\hbar \alpha T_{\mathrm{LZ}} / 2 \delta\right) \\
\sin \left(\hbar \alpha T_{\mathrm{LZ}} / 2 \delta\right) & \cos \left(\hbar \alpha T_{\mathrm{LZ}} / 2 \delta\right)
\end{array}\right)
$$

with

$$
\sin ^{2}\left(\frac{\hbar \alpha}{2 \delta} T_{\mathrm{LZ}}\right)=P_{\mathrm{LZ}}
$$

In the next section we will discuss how to apply this model for LZ transitions to the problem of interband transitions in a sinusoidal lattice.

\section{Interband tunneling in a lattice}

We are interested in describing the tunneling process from the first to the second band of a Bose-Einstein condensate trapped in an optical lattice. We assume that almost all atoms are in the condensate, so that the system is described by a single-particle wave function $\psi(x, t)$ [17]. Moreover, let the condensate be dilute enough so that the interaction between particles can be neglected. This implies that the wave function of the system obeys a linear Schrödinger equation.

The experimental condition is that of an accelerating one-dimensional optical lattice, with constant acceleration $a$ [18]. In the rest frame of the lattice, a particle 
of mass $m$ in the lattice is subjected to an external force $F=m a$ and a potential $(V / 2) \cos \left(2 \pi x / d_{L}\right), V$ being the lattice depth and $d_{L}$ the lattice period (half wavelength of the counterpropagating laser beams). The dynamics of the system depends on two dimensionless parameters [19], related to lattice depth and external force:

$$
V_{0}=\frac{V}{E_{\text {rec }}}, \quad F_{0}=\frac{F d_{L}}{E_{\text {rec }}}, \quad \text { with } \quad E_{\text {rec }}=\frac{\hbar^{2}}{2 m}\left(\frac{\pi}{d_{L}}\right)^{2} .
$$

In the adiabatic approximation, no transition takes place between bands (single-band approximation). This is consistent if $F d_{L} \lesssim V$, namely if $F_{0} \lesssim V_{0}$ in Eq. (19), and leads us to the two-level approximation outlined in the previous section.

We are interested in experimental setups in which the initial state is highly peaked around a single quasimomentum value $k_{0}$, that is, the width of the initial quasimomentum distribution is much smaller than the width $2 \pi / d_{L}$ of the first Brillouin zone $\mathcal{B}$. Under this condition, in the adiabatic single-band approximation, the average quasi-momentum evolves semiclassically [16, 20], so that at time $t$

$$
k(t)=k_{0}+\frac{F t}{\hbar}
$$

with negligible spread in the distribution occurring during the evolution. This yields Bloch oscillations in a tilted lattice with a Bloch period

$$
T_{\mathrm{B}}=\frac{2 \pi \hbar}{F d_{L}}=\frac{\hbar}{E_{\mathrm{rec}}} \frac{2 \pi}{F_{0}} .
$$

The initial state analyzed here has a well defined initial momentum (in $\mathcal{B}$ ), but can be distributed among different bands. At the end of each Bloch period, the amplitude in band $\alpha$ acquires the following phase with respect to the amplitude in band $\beta$

$$
\Delta \varphi_{\alpha \beta}=-\frac{2 \pi}{F_{0}}\left\langle E_{\alpha}(k)-E_{\beta}(k)\right\rangle,
$$

where $\langle\ldots\rangle$ denotes the average over $\mathcal{B}$ and $E_{\gamma}(k)$ is the energy of the state with quasimomentum $k$ in band $\gamma$ in units $E_{\text {rec }}$. The relative phases acquired by states in different bands are at the origin of Stückelberg oscillations in the interband transition rates [14].

If the initial quasimomentum distribution is very peaked around its central value, the interband transition can be analyzed as a Landau-Zener tunneling, since in suitable parameter ranges the transition from the first to the second band is concentrated at the edges of the Brillouin zone, where an avoided crossing occurs. In the Pisa experiment analyzed here [12], this leads to an alternation of plateaus and steep transition regions of the survival probability in the first band (see Fig. 2). The diabatic basis is represented by the momentum eigenstates, crossing at $\hbar \pi / d_{L}$ and coupled with strength $V / 4$, while the adiabatic basis is represented by the quasimomentum eigenstates. The LZ parameters in Eq. (15) are

$$
\alpha=\frac{\pi \hbar F}{m d_{L}}, \quad \delta=\frac{V}{4},
$$


yielding the transition probability 18

$$
P_{\mathrm{LZ}}^{(1,2)}\left(V_{0}, F_{0}\right)=\exp \left(-\frac{\pi^{2} V_{0}^{2}}{32 F_{0}}\right) .
$$

Even if the essential features of the transition are included in Eq. (24), discrepancies can arise between the idealized case and the real physical situation. Indeed, the periodicity of the lattice implies that the afore-mentioned process occurs in a finite time, and that in the initial and final states the adiabatic levels are not infinitely separated. The corrections to the LZ transition probability due to the finite duration of the process are discussed in [20, 19]. Other corrections to Eq. (24) should be considered if the lattice is not shallow. In this case, couplings to higher momentum states play an important role and a two-level description is no longer a good approximation.

Moreover, there is another kind of deviation from LZ, which is the main object of our analysis. Since Eq. 24 is obtained under the hypothesis that only one of the two adiabatic states is initially populated, it is no longer valid if both states are populated. These deviations can be relevant even if one of the initial populations is very close to zero, since they scale as the square root of the smallest population, as will be discussed in the following. These interference effects lead to resonantly enhanced tunneling (RET): the transition probability is enhanced by a large factor with respect to the LZ prediction if the energy difference $F d_{L} \Delta i$ between two potential wells $\left(d_{L}\right.$ being the lattice spacing and $d_{L} \Delta i$ the distance between the wells) matches the average band gap of the non-tilted system.

The dynamics of interband tunneling can be schematized by separately describing the transition at the avoided crossing and the evolution far from the edges of the Brillouin zone. We will assume that the transition between the first and the second band occurs in a time that is negligible with respect to the Bloch time defined in (21), namely $T_{\mathrm{LZ}} \ll T_{B}$. We are thus assuming that the evolution inside the first band is adiabatic for all $k$, except for $k \simeq \pi / d_{L}$, when a transition towards the state with the same quasi-momentum in the second band is possible.

This transition can be effectively described by an evolution operator corresponding to the one defined in Eq. (17):

$$
\tilde{U}=\left(\begin{array}{cc}
s_{12} & -p_{12} \\
p_{12} & s_{12}
\end{array}\right),
$$

with $s_{12}=\sqrt{P_{\mathrm{LZ}}\left(V_{0}, F_{0}\right)}$ and $p_{12}=\sqrt{1-s_{12}^{2}}$. The operator $\tilde{U}$ acts on the twodimensional space spanned by $\{|1\rangle,|2\rangle\}$, the states with the same quasimomentum in the first and the second band, respectively, that evolve according to 20 with $k_{0}=0$.

The transition from the second to the third band can be schematized as the loss of a fraction $1-s_{23}^{2}$ in the population of the second band towards a continuum, occurring at the crossing around $k=0$. This assumption is justified for small values of $V_{0}$, such that a particle in the third (or higher) band can be considered free. The survival amplitude $s_{23}$ can be determined by imposing that its square be equal to $1-P_{\mathrm{LZ}}^{(2,3)}\left(V_{0}, F_{0}\right)$, where 
$P_{\mathrm{LZ}}^{(2,3)}\left(V_{0}, F_{0}\right)$ the $\mathrm{LZ}$ transition probability at the avoided crossing from the second to the third band

$$
P_{\mathrm{LZ}}^{(2,3)}\left(V_{0}, F_{0}\right)=\exp \left(-\frac{\pi^{2} V_{0}^{4}}{2^{14} F_{0}}\right) .
$$

During each Bloch cycle separating two successive transitions, the relative phase between the second and the first band amplitudes increases by the quantity 22 , that reads

$$
\phi\left(V_{0}, F_{0}\right)=-\frac{2 \pi}{F_{0}}\left\langle\Delta E\left(V_{0}\right)\right\rangle,
$$

where $\langle\Delta E\rangle$ is the energy difference (in units $E_{\text {rec }}$ ) between the second and the first band, averaged over $\mathcal{B}$. The effects of the dynamics in a time $T_{\mathrm{B}}$ from one transition to the next one can thus be modelled in the basis $\{|1\rangle,|2\rangle\}$ by an effective non-unitary operator

$$
W=\left(\begin{array}{cc}
1 & 0 \\
0 & s_{23} \mathrm{e}^{i \phi}
\end{array}\right) .
$$

The lack of unitarity is due to the two-level approximation adopted in our analysis and signals the flow of population out of the relevant two-dimensional subspace. Observe that the global evolution of the condensate is always coherent and does not involve any atomic losses.

By making use of this simplified model, we describe the time evolution in the following way. At $t=0$ the condensate is in the first band, with quasi-momentum close to $k=0$. As the lattice is accelerated, the quasi-momentum increases until it reaches $\pi / d_{L}$ at $t=T_{\mathrm{B}} / 2$, where the operator $\tilde{U}$ comes into play and transfers part of the population to the second band. The evolution from $T_{\mathrm{B}} / 2$ to $3 T_{\mathrm{B}} / 2$ is summarized by the application of $W$. Then, the second transition occurs, and part of the population in the second band (minus losses towards the third band) can tunnel back to the first band due to the action of $\tilde{U}$, giving rise to interference effects. The same steps occur in the subsequent transitions.

On a time span $T_{\mathrm{B}}$, the dynamics of the system is determined by the action of the non-unitary operator

$$
U=\tilde{U} W=\left(\begin{array}{cc}
s_{12} & -p_{12} s_{23} \mathrm{e}^{i \phi} \\
p_{12} & s_{12} s_{23} \mathrm{e}^{i \phi}
\end{array}\right)
$$

in the basis $\{|1\rangle,|2\rangle\}$. The order of the two operations is not relevant, since $W$ acts trivially on the initial state $|1\rangle$ before the first transition.

\section{Transient and asymptotic dynamics}

We now look at the time evolution evolution implied by the model outlined in the preceding section. The state of the system before the first transition is $|1\rangle$. Immediately after the $n$-th transition, occurring at time $t=T_{\mathrm{B}}(n+1 / 2)$, the state of the system is

$$
\left|\Phi_{n}\right\rangle=U^{n}|1\rangle \text {. }
$$


Let $\psi_{1}$ and $\psi_{2}$ be the normalized non-orthogonal eigenvectors of the matrix $U$ belonging to the eigenvalues $e_{1}$ and $e_{2}$, respectively. The initial state

$$
|1\rangle=c_{1}\left|\psi_{1}\right\rangle+c_{2}\left|\psi_{2}\right\rangle \text {, }
$$

will evolve at time $T_{\mathrm{B}}(n+1 / 2)$ into

$$
\left|\Phi_{n}\right\rangle=c_{1} e_{1}^{n}\left|\psi_{1}\right\rangle+c_{2} e_{2}^{n}\left|\psi_{2}\right\rangle .
$$

Due to the non-unitarity of $W$, the two eigenvalues are smaller than unity. Let us assume that the eigenvalue $e_{1}$ is larger in modulus than $e_{2}$, so that it eventually dominates in the iteration (32). (This condition is necessarily verified for $0<s_{12}, s_{23}<1$.) For $n$ sufficiently large, the evolution reaches an asymptotic regime, in which the state after the $n$-th transition is determined only by the state after the previous one, with a transition rate depending on the largest eigenvalue. Since the survival probability in the first band can be defined as $P_{n}=\left|\left\langle 1 \mid \Phi_{n}\right\rangle\right|^{2}$, in the asymptotic regime one gets

$$
P_{n} \simeq\left|e_{1}\right|^{2} P_{n-1} \text {. }
$$

We define the asymptotic transition rate

$$
\gamma=-\ln \left(\left|e_{1}\right|^{2}\right)
$$

and introduce an exponential function $P_{\mathrm{Z}}(t)$ that coincides with the survival probability at the center of the plateaus, at times $t=n T_{\mathrm{B}}$ :

$$
P_{\mathrm{Z}}(t)=Z \exp (-\gamma t) \text {. }
$$

This is the dashed line plotted in Fig. 2. (Note the logarithmic scale on the ordinates.)

Observe that Eqs. (33)- 35) are valid in the asymptotic regime. Before reaching it, the ratio between $P_{n}$ and $P_{n-1}$ in Eq. (28) depends on $n$ through a time-dependent decay rate $\gamma_{n}$, as in

$$
P_{n+1}=\mathrm{e}^{-\gamma_{n}} P_{n}
$$

It is easy to prove that the succession $\gamma_{n}$ converges to the value $\gamma$ of Eq. (34), unless the eigenvalues of $U$ have equal moduli. Indeed

$$
\gamma_{n}=-\ln \frac{P_{n+1}}{P_{n}}=-\ln \left|\frac{c_{1} e_{1}^{n+1}\left\langle 1 \mid \psi_{1}\right\rangle+c_{2} e_{2}^{n+1}\left\langle 1 \mid \psi_{2}\right\rangle}{c_{1} e_{1}^{n}\left\langle 1 \mid \psi_{1}\right\rangle+c_{2} e_{2}^{n}\left\langle 1 \mid \psi_{2}\right\rangle}\right|^{2},
$$

which, by definition of $\gamma$ in Eq. (34), reads

$$
\gamma_{n}=\gamma-2 \ln \left|\frac{1+\frac{c_{2}\left\langle 1 \mid \psi_{2}\right\rangle}{c_{1}\left\langle 1 \mid \psi_{1}\right\rangle}\left(\frac{e_{2}}{e_{1}}\right)^{n+1}}{1+\frac{c_{2}\left\langle 1 \mid \psi_{2}\right\rangle}{c_{1}\left\langle 1 \mid \psi_{1}\right\rangle}\left(\frac{e_{2}}{e_{1}}\right)^{n}}\right| .
$$

Thus, if $\left|e_{2}\right|<\left|e_{1}\right|$, the argument in the logarithm approaches one and $\gamma_{n} \rightarrow \gamma$. 


\section{Wave function renormalization}

The parameter $Z$ in Eq. (35) is in general different from unity, due to the transient regime at the beginning of the evolution. It represents the extrapolation of the asymptotic exponential probability back at $t=0$. We derive below an expression for $Z$. In the asymptotic regime, the system evolution described by Eq. (32) corresponds to an evolution operator applied to an initial unnormalized vector $\left|\Psi_{0}\right\rangle=c_{1}\left|\psi_{1}\right\rangle$ :

$$
\left|\Phi_{n}\right\rangle \simeq c_{1} e_{1}^{n}\left|\psi_{1}\right\rangle=U^{n}\left(c_{1}\left|\psi_{1}\right\rangle\right)=U^{n}\left|\Psi_{0}\right\rangle
$$

The $Z$ parameter, representing the extrapolation of the asymptotic behavior back to $t=0$, can be defined as the square modulus of the projection of the fictitious initial vector $\left|\Psi_{0}\right\rangle$, onto the actual initial state $|1\rangle$

$$
Z \equiv\left|\left\langle 1 \mid \Psi_{0}\right\rangle\right|^{2}=\left|c_{1}\right|^{2}\left|\left\langle 1 \mid \psi_{1}\right\rangle\right|^{2},
$$

which corresponds to an extrapolated "survival probability" in the subspace spanned by $|1\rangle$, evaluated at the initial time. In order to gain a qualitative understanding of the dependence of $Z$ on the phase difference $\phi$ acquired during a Bloch cycle, let us compare the first and second transitions. Let $P_{0}=1$ be the initial value of the survival probability in the first band. After the first transition, application of Eq. (29) yields the survival probability

$$
P_{1}=s_{12}^{2} P_{0} \equiv \mathrm{e}^{-\gamma_{0}} P_{0} .
$$

At the second transition, the discrepancy with the LZ prediction becomes manifest. In the parameter regime of small $V_{0}$ we are considering, the ratio $s_{23} / s_{12}$ is very small [12] and we can apply a first-order approximation, yielding

$$
P_{2} \simeq\left(s_{12}^{2}-2 s_{23} p_{12}^{2} \cos \phi\right) P_{1} \equiv \mathrm{e}^{-\gamma_{1}} P_{1} .
$$

This clarifies that the plateaus in Fig. 2 are not equal. If the phase is $\phi=2 \pi j$, with integer $j$, the second transition is enhanced with respect to the first one. Thus, comparing with Eq. (27), local maxima in the transition rate are expected when $F_{0}(\phi=2 \pi j)=\left\langle\Delta E\left(V_{0}\right)\right\rangle / j$.

This is the mechanism at the origin of wave function renormalization. A backwards extrapolation of the second step gives a rough estimate of the $Z$ parameter, which we call $Z_{1}$ :

$$
Z_{1}=\exp \left(\gamma_{1}-\gamma_{0}\right) \simeq 1+2 s_{23}\left(\frac{p_{12}}{s_{12}}\right)^{2} \cos \phi .
$$

Even if Eq. (43) represents a rather crude approximation, it is very useful in an experimental context, where only the first few steps in the Bloch cycles are accessible. If the survival amplitude can be measured up to the $N$-th transition, the $Z$ parameter can be approximated by

$$
Z_{N}=\exp \left(N \gamma_{N}-\sum_{n=0}^{N-1} \gamma_{n}\right) \rightarrow Z
$$




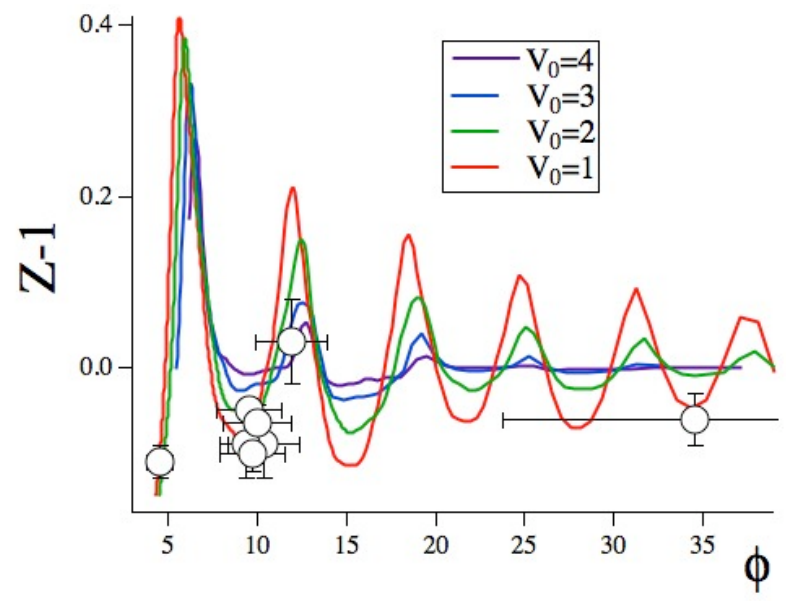

Figure 3. Scaling plot of $Z-1$ vs. $\phi$ of Eq. (27), derived from RET experimental results obtained in Ref. [12] (open circles) using a narrow atomic quasi-momentum distribution. The full lines are the theoretical predictions for $V_{0}=1,2,3,4$ (a smaller value of $V_{0}$ yielding wider oscillations).

where the convergence to a fixed asymptotic value $Z$ is due to (38). The convergence to $Z$ is typically very fast, and the first few cycles are already sufficient to obtain an excellent approximation. $Z$ is displayed in Fig. 3 as a function of $\phi$.

\section{Conclusions}

In this article we studied Landau-Zener transitions and used them as a benchtest for the study of wave-function renormalization effects in quantum decay processes. By scrutinizing the features of the survival probability of the wave function that collectively describes an ultra-cold atomic cloud, we consistently defined $Z$ and extracted information on its behavior as a function of the experimental parameters.

The value of $Z$ reflects the overlap between a discrete state and a continuum of states (to which the discrete state decays). Pictorially, one might say that $Z$ detects the overlap $Z=\left|\left\langle\psi_{\mathrm{G}} \mid \psi_{\text {in }}\right\rangle\right|^{2}$ between the (generalized) decaying eigenfunction with complex energy eigenvalue, the Gamow state $\psi_{\mathrm{G}}$, and the initial (physical) state $\psi_{\text {in }}$. The case $Z<1$ does not surprise, being in accord with the familiar Källén-Lehmann paradigm [2] for stable asymptotic states. The situation $Z>1$ is more curious, and is a consequence of the fact that $\psi_{\mathrm{G}}$ does not live in the Hilbert space, its norm being infinite [5, 6, 7], and its overlap $Z$ with the initial state can exceed 1 .

It is remarkable that a quantity like $Z$ can be directly measured and that its deviations from unity yields directly measurable consequences. In addition, as the experimental parameters are varied, $Z$ can take values that can be smaller or larger than unity. 
Unearthing wave-function renormalization effects in the time evolution of a Bose-Einstein condensate12

\section{Acknowledgments}

We thank G. Florio, H. Lignier, N. Lörch, R. Mannella and S. Wimberger for many

interesting discussions. The experiment discussed in this article was done in Pisa. The numerical analyses in Figs. 2 and 3 were performed by N. Lörch and S. Wimberger.

\section{References}

[1] S. Weinberg, "The Quantum Theory of Fields: Volume I Foundations" (Cambridge University Press, 1995); M. Peskin and D. Schoeder, "An Introduction to Quantum Field Theory" (Perseus Books Group, 1995).

[2] G. Källén, Helv. Phys. Acta 25, 417 (1952); H. Lehmann, Nuovo Cim. 11, 342 (1954).

[3] H. Nakazato, M. Namiki and S. Pascazio, Int. J. Mod. Phys. B10, 247 (1996).

[4] L. S. Brown, Quantum Field Theory (Cambridge University Press, Cambridge, 1994).

[5] G. Gamow, Z. Phys. 51, 204 (1928).

[6] A. Bohm, Quantum Mechanics: Foundations and Applications (Springer, Berlin, 1994); A. Bohm, M. Gadella, and B. Mainland, Am. J. Phys. 57, 1103 (1989).

[7] I. Antoniou, M. Gadella, J. Mateo and G.P. Pronko, Int J. Theor. Phys. 42, 2389 (2003); O. Civitarese and M. Gadella, Phys. Rept. 396, 41 (2004); M. Gadella and G.P. Pronko, Fortschr. Phys. 59, 795 (2011); R. de la Madrid, J. Math. Phys. 53, 102113 (2012).

[8] Wilkinson S R, Bharucha C F, Fischer M C, Madison K W, Morrow P R, Niu Q, Sundaram B and Raizen M G 1997 Nature $\mathbf{3 8 7} 575$

[9] Fischer M C, Gutiérrez-Medina B and Raizen M G 2001 Phys. Rev. Lett. 87040402

[10] S. Pascazio and P. Facchi, Acta Phys. Slovaca 49, 557 (1999); Phys. Rev. A62, 023804 (2000); Phys. Rev. Lett. 86, 2699 (2001).

[11] A. G. Kofman and G. Kurizki, Acta Phys. Slovaca 49, 541 (1999); Nature 405, 546 (2000).

[12] N. Lörch, F. V. Pepe, H. Lignier, D. Ciampini, R. Mannella, O. Morsch, E. Arimondo, P. Facchi, G. Florio, S. Pascazio and S. Wimberger, Phys. Rev. A85, 053602 (2012).

[13] L. Landau, Phys. Z. Sowjetunion 2, 46 (1932); C. Zener, Proc. R. Soc. London, Ser. A 137, 696 (1932).

[14] E. C. G. Stückelberg, Helv. Phys. Acta 5, 369 (1932).

[15] E. Majorana, Nuovo Cimento 9, 43 (1932).

[16] H. Jones and C. Zener, Proc. R. Soc. 144, 101 (1934).

[17] L. Pitaevskii, and S. Stringari, Bose-Einstein Condensation (Clarendon Press, Oxford, 2003).

[18] A. Zenesini, H. Lignier, G. Tayebirad, J. Radogostowicz, D. Ciampini, R. Mannella, S. Wimberger, O. Morsch, E. Arimondo, Phys. Rev. Lett. 103, 090403 (2009).

[19] G. Tayebirad, A. Zenesini, D. Ciampini, R. Mannella, O. Morsch, E. Arimondo, N. Lörch, and S. Wimberger, Phys. Rev. A 82, 013633 (2010).

[20] M. Holthaus, J. Opt B: Quantum Semiclass. Opt. 2, 589 (2000). 